\title{
Correction to: Properties of Asphalt Binders with Increasing SBS Polymer Modification
}

\author{
Mike Aurilio, Peter Mikhailenko, Hassan Baaj, \\ and Lily D. Poulikakos
}

\begin{abstract}
Correction to:
Chapter "Properties of Asphalt Binders with Increasing SBS Polymer Modification" in: M. Pasetto et al. (Eds.):

Proceedings of the 5th International Symposium on Asphalt

Pavements \& Environment (APE), LNCE 48, https://doi.org/10.1007/978-3-030-29779-4_6
\end{abstract}

In the original version of the book, the author name "Mike Aurillio" has been changed to "Mike Aurilio" in the Frontmatter, Backmatter and Chapter 6. The correction chapter and book have been updated with the changes. 